\title{
Toxina botulínica A e repercussões na capacidade para andar de indivíduos pós acidente
} vascular cerebral: revisão sistemática

\section{Botulinum toxin A and repercussions on the ability to walk in individuals after a stroke: systematic review}

\author{
Layane Mendes Alves ${ }^{1}$, Lucas dos Santos Galaverna ${ }^{1}$, (iD Lilian de Fátima Dornelas ${ }^{2}$
}

\begin{abstract}
RESUMO
A indicação da toxina botulínica do tipo A (TBA) para indivíduos com história de acidente vascular cerebral (AVC) é uma prática clínica comum para redução da espasticidade. Objetivo: Investigar se a ação da TBA sem associação com outras intervenções impacta na capacidade da atividade de andar nos indivíduos pós-AVC. Método: Revisão sistemática com estudos provenientes da Cochrane Central Register of Controlled Trials, MEDLINE, SciELO, PEDro e LILACS, em inglês e português, entre 2010 a 2020. Foram incluídos ensaios clínicos controlados, com participantes pós AVC submetidos a aplicação de TBA em idades entre 18 a 50 anos de ambos os sexos. A seleção dos artigos foi realizada por dois avaliadores, com extração dos dados e avaliação de qualidade da evidência pela PEDro, segundo critérios de inclusão e exclusão pré-estabelecidos. Resultados: Dos 183 artigos, quatro permaneceram para análise final. A aplicação da TBA em indivíduos pós AVC ocorreu em sua maioria em flexores plantares e os achados relacionados com a atividade andar foram o aumento da passada, da velocidade da marcha, de distâncias percorridas ao caminhar e redução do tempo de execução de ações, como subir e descer degraus. Conclusão: A TBA proporciona melhora da capacidade para andar em indivíduos pós AVC e o conhecimento dos seus benefícios pós aplicação é essencial para informar aos pacientes da importância de aproveitar este momento, para modificar comportamentos que acentuam os padrões de compensação, que tanto corroboram para o retorno de limitações de atividades e não devido ao término da ação da TBA.
\end{abstract}

Palavras-chaves: Acidente Vascular Cerebral, Espasticidade Muscular, Marcha, Toxinas Botulínicas Tipo A

\section{ABSTRACT}

The indication of botulinum toxin type A (BoNTA) for individuals with a history of stroke is a common clinical practice for reducing spasticity. Objective: To investigate whether the action of TBA without association with other interventions impacts on the ability of walking activity in post-stroke individuals. Method: A systematic review with studies from the Cochrane Central Register of Controlled Trials, MEDLINE, SciELO, PEDro, and LILACS databases, in English and Portuguese, between 2010 and 2020. Controlled clinical trials were included, with post-stroke participants aged between 18 and 50, of both sexes, submitted themselves to the application of BoNTA. The selection of the articles was carried out by two evaluators, with data extraction and quality evaluation of the evidence by PEDro, according to pre-established inclusion and exclusion criteria. Results: Of the 183 articles, four remained for final analysis. The application of TBA in post-stroke individuals occurred mostly in plantar flexors and the findings related to walking activity were increased stride, gait speed, distances covered when walking and reduced time to perform actions, such as climbing and go down steps. Conclusion: TBA improves the ability to walk in post-stroke individuals and knowledge of its benefits after application is essential to inform patients of the importance of taking advantage of this moment, to modify behaviors that accentuate the compensation patterns, which so much corroborate for the return of activity limitations and not due to the termination of the TBA action.

Keywords: Stroke, Muscle Spasticity, Gait, Botulinum Toxins, Type A 


\section{INTRODUÇÃO}

O Acidente Vascular Cerebral (AVC) é uma doença cerebrovascular que corresponde a segunda maior causa de morte no mundo. ${ }^{1}$ No Brasil, estima-se cerca de 400 mil novos casos por ano, sendo estes responsáveis por cerca de $100 \mathrm{mi}$ mortes no país. ${ }^{2}$ Embora a taxa de mortalidade para o AVC seja alta, o índice de morbidade também é elevado, acarretando incapacidades decorrentes dos problemas estruturais e funcionais do corpo, limitações de atividades e restrições sociais, o que impacta diretamente na qualidade de vida destes indivíduos. ${ }^{3-5}$

Dentre as incapacidades advindas do AVC, a espasticidade que é uma alteração da função do corpo e está entre os principais problemas da função corporal ocorrendo em cerca de $90 \%$ dos casos no hemicorpo geralmente contralateral à lesão cerebral, com uma prevalência que varia entre $4 \%$ a $27 \%$ nas primeiras 6 semanas após o AVC. ${ }^{6,7}$ Este prejuízo da função e estrutura do corpo pode trazer incapacidades e comprometer a atividade de andar, que compreende a capacidade, que é definida como uma tarefa ou ação por um indivíduo. ${ }^{8}$ Neste contexto, podem ocorrer dificuldades na execução de atividades, como andar, subir ou descer degraus, realizar atividades domésticas e do dia a dia, por exemplo.

A espasticidade é compreendida como um aumento da atividade neuronal, que causa aumento do tônus muscular velocidade dependente, exacerbação de reflexos, ocasionando dor e possíveis contraturas articulares, se não tratada. ${ }^{9}$ A espasticidade advinda após o AVC envolve tanto vias neurais aferentes quanto eferentes e interneurônios medulares, com origem na interrupção inibitória sobre os neurônios motores alfa e gama, os quais sofrerão aumento da excitabilidade. ${ }^{10} \mathrm{O}$ efeito da espasticidade pós AVC acaba gerando perda de controle de movimentos, espasmos dolorosos, perda do padrão postural normal e redução das funções musculares, incluindo de membros inferiores, ${ }^{11}$ o que impacta diretamente na capacidade da marcha desses indivíduos.

Um dos tratamentos para a espasticidade é o uso local da Toxina Botulínica tipo A (TBA). ${ }^{12}$ A TBA é sintetizada pelo Clostridium Botulinum, uma bactéria anaeróbica ${ }^{13,14} \mathrm{e}$ apresenta diferentes tipos sorológicos, sendo o tipo A o mais potente e utilizado na prática clínica. A ação consiste no bloqueio de liberação de substâncias nociceptoras no neurônio pré-sináptico, reduzindo a hiperexcitabilidade do neurônio pós sináptico através, também, da degradação de proteínas que realizam a fusão de vesículas com este neurotransmissor à membrana neuronal.

Consequentemente, ocorre a redução da concentração de acetilcolina na fenda sináptica, bloqueando a ativação excitatória de contração muscular ${ }^{15-17}$ com efeito transitório e reversível após alguns meses de sua administração. ${ }^{14}$ Sua aplicação é realizada de forma intramuscular com dosagem e volume que são determinados pelo médico, o qual prescreve de acordo com a clínica do indivíduo, objetivo e tratamento. Além disso, cada músculo tem uma dose média (mínima e máxima) e para delimitar o ponto de injeção é importante seguir o guia e o mapa de pontos motores. ${ }^{13,14}$

A TBA tem sido indicada amplamente para tratar a espasticidade de adultos pós AVC. ${ }^{18}$ Karadag-Saygi et al. ${ }^{19} \mathrm{em}$ uma análise retrospectiva aplicaram a TBA em indivíduos pós
AVC que apresentavam espasticidade em membros superiores e inferiores e verificaram que a TBA enfraqueceu seletivamente a musculatura, facilitando a realização de atividades de vida diária de maneira mais independente. Além disso, estudos ${ }^{20}$ apontam que ao associar a TBA com a fisioterapia os benefícios são prolongados.

\section{OBJETIVO}

É consenso na literatura que a ação da TBA reduz a espasticidade muscular de maneira efetiva, contudo, o presente estudo pretende investigar se sua ação sem associação com outras intervenções impacta na capacidade da atividade de andar nos indivíduos pós-AVC.

\section{MÉTODOS}

Este estudo se caracteriza por uma revisão sistemática da literatura em que seguiu as recomendações PRISMA Statement. ${ }^{21}$ A seleção de estudos pertinentes ao tema teve como pergunta norteadora: Há mudanças no desempenho da atividade andar de indivíduos pós AVC que recebem aplicação isolada da TBA? Onde pelo acrônimo PICOT:

\section{$P$ - População: AVC \\ I - Intervenção: TBA \\ C - Comparação: não se aplica \\ O - Desfecho: atividade andar \\ $T$ - Estudos: ensaios clínicos, estudos clínicos randomizados}

Os termos foram selecionados de acordo com os descritores em Ciências da Saúde (DESC), na língua portuguesa: Acidente Vascular Cerebral; Espasticidade muscular; Hemiplegia e na língua inglesa: Stroke; Muscle Spasticity; Hemiplegia. Para a estratégia de busca foram utilizados os operadores boleanos OR e AND e as bases de dados para esta busca foram: Cochrane Central Register of Controlled Trials, MEDLINE, SciELO, PEDro e LILACS, utilizando termos nos idiomas inglês e português, realizados entre os anos de 2010 a março de 2020, além da busca manual nas referências dos artigos selecionados, conforme é demonstrado no Quadro 1.

A busca de artigos foi realizada no período de fevereiro a abril de 2020 e a seleção dos estudos foi realizada por dois avaliadores independentes correspondendo aos seguintes critérios:

Critérios de inclusão: indivíduos pós-AVC que foram submetidos a aplicação da TBA em membros inferiores com idades entre 18 a 50 anos de ambos os sexos.

Critérios de exclusão: estudos que abordavam membros superiores; terapias combinadas além da aplicação da TBA, como exercícios físicos; estudos de caso e revisões; estudos que não tiveram como desfecho a capacidade da atividade de andar, que é definida como uma tarefa ou ação por um indivíduo.

Para a extração dos dados foram tabuladas informações dos estudos sobre: autor/ano, objetivo, metodologia, resultados e conclusão e também foi realizada a avaliação de qualidade metodológica por meio da escala PEDro. ${ }^{22} \mathrm{~A}$ escala PEDro é utilizada para investigar a eficácia de intervenções em fisioterapia, em que todos os estudos controlados aleatorizados são avaliados em sua descrição metodológica e 
estatística em 11 pontos de mensuração, com base nos seguintes critérios: 1- Especificação dos critérios de elegibilidade; 2- Aleatorização dos sujeitos do estudo; 3alocação dos sujeitos secreta; 4- Inicialmente, os grupos eram semelhantes aos indicadores de prognóstico mais importante; 5- Todos os sujeitos participaram de forma cega no estudo; 6 Todos os terapeutas do estudo participaram de forma cega; 7Os avaliadores que fizeram medição dos resultados chave eram cegos; 8- Mensuração de pelo menos um resultado chave obtido em mais de $85 \%$ dos sujeitos inicialmente distribuídos pelos grupos; 9- Todos os sujeitos receberam tratamentos ou condição de controle conforme alocação, ou quando não foi esse o caso, fez-se análise dos dados para pelo menos um dos resultados chave por "intenção de tratamento"; 10- Os resultados das comparações estatísticas intergrupos foram descritos para pelo menos um resultado chave; e 11- 0 estudo apresentou medidas de precisão e medidas de variabilidade de ao menos um resultado chave.

É importante ressaltar que o uso da escala PEDro não avalia a interpretação dos resultados das intervenções, e sim quanto a qualidade metodológica do estudo. ${ }^{22}$ Com isso, para esta revisão foram selecionados estudos que obtiveram notas superiores ou iguais a 7 na escala, afim de se obter ensaios clínicos com boa qualidade metodológica.

Quadro 1. Termos utilizados e números de artigos encontrados e selecionados nas bases de dados

\begin{tabular}{|c|c|c|c|}
\hline Bases de dados & Termos utilizados & $\begin{array}{c}\text { Artigos } \\
\text { Encontrados }\end{array}$ & $\begin{array}{c}\text { Artigos } \\
\text { Selecionados }\end{array}$ \\
\hline Medline (PubMed) & $\begin{array}{l}\text { "Botulinum Toxin Type A"[All Fields] AND } \\
\text { (("stroke"[MeSH Terms] OR "stroke"[All Fields]) AND } \\
\text { ("muscle spasticity"[MeSH Terms] OR ("muscle"[All } \\
\text { Fields] AND "spasticity"[All Fields]) OR "muscle } \\
\text { spasticity"[All Fields] OR "spasticity"[All Fields])) AND } \\
\text { ("2010/02/21"[PDat]:"2020/02/18"[PDat]) }\end{array}$ & 134 & 1 \\
\hline Biblioteca Virtual em Saúde (BVS) & Botulinum Toxin Type A and stroke and spasticity & 3 & 2 \\
\hline PEDro & botulinum toxin and stroke & 22 & 0 \\
\hline Cochrane & botulinum toxin and stroke & 3 & 0 \\
\hline Scielo & Botulinum Toxin Type A and spasticity & 21 & 1 \\
\hline
\end{tabular}

\section{RESULTADOS}

Quatro estudos ${ }^{18,23,24,25}$ foram selecionados para a análise final da revisão, como mostra a Figura 1.

Os estudos desta revisão (Quadro 2) foram compostos por indivíduos adultos, em sua maioria do sexo masculino, com até 2 anos pós AVC, hemiparéticos, que receberam a aplicação da TBA preferencialmente no tríceps sural. A seleção dos grupos musculares, dos pontos para aplicação e das doses da TBA foram comumente definidas por critério médico, após avaliação clínica de cada caso, levando em conta o grau de espasticidade.

Com relação aos instrumentos de avaliação mais utilizados para a mensuração antes e depois da intervenção destacam-se o teste de caminhada de 10 metros (10-TWM), a Escala Modificada de Ashwoth (MAS), o Teste de caminhada de 6 minutos (TC6M), o Time up and go (TUG), o Teste do degrau para avaliar tríplice flexão de MMII, a Escala de Fulg Meyer e a Escala de Realização de Objetivos (GAS).

Quanto aos principais efeitos da TBA na atividade andar foram encontrados o aumento da velocidade ao caminhar, maiores distâncias percorridas, redução do tempo de execução de atividades como: subir e descer escadas e aumento da amplitude de movimento da dorsiflexão na fase de balanço durante na marcha em indivíduos pós AVC.

As reavaliações do efeito da aplicação da TBA foram realizadas em sua maioria, no período de 2 a 12 semanas.

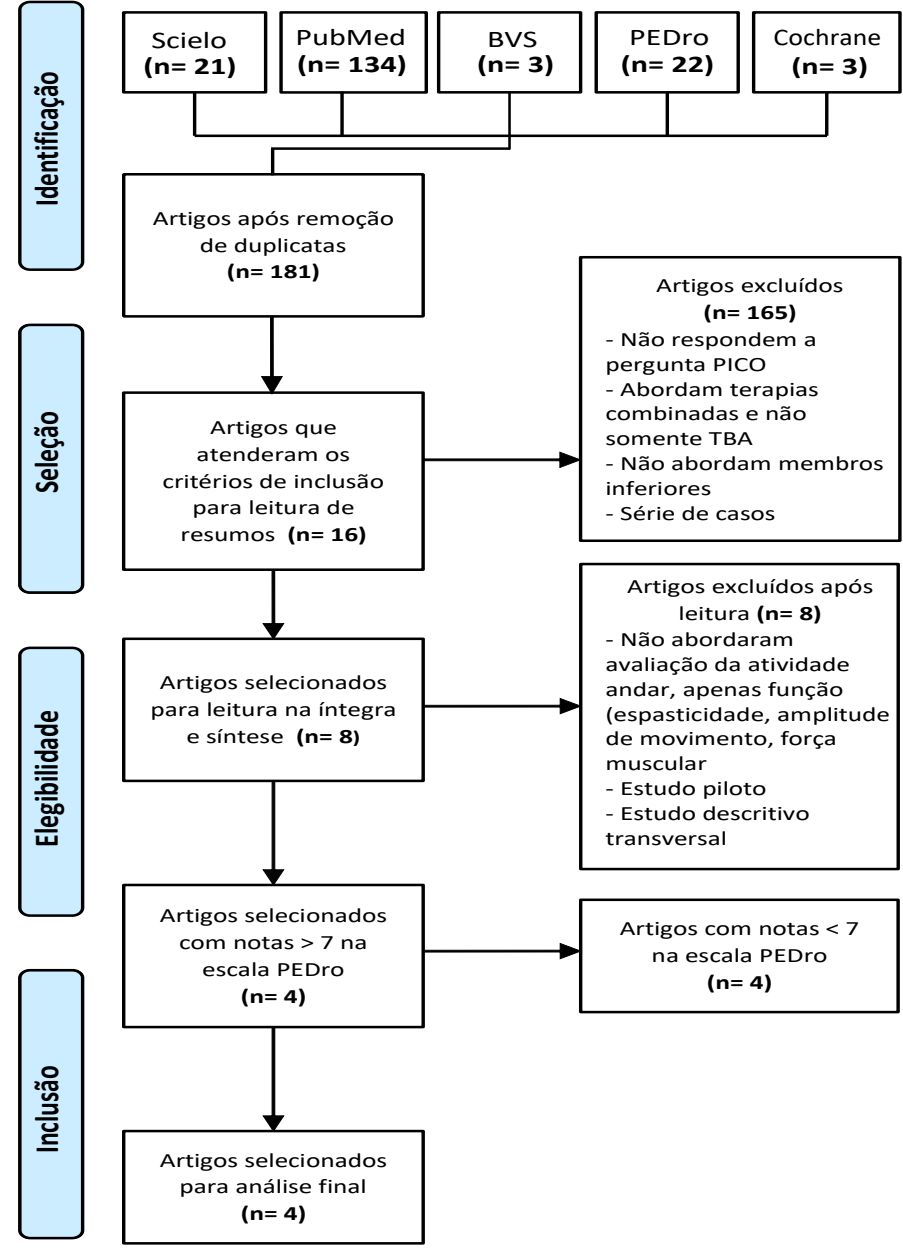

Figura 1. Fluxograma das diferentes fases da revisão sistemática 
Quadro 2: Caracterização dos artigos selecionados sobre repercussões na marcha após aplicação de toxina botulínica tipo A

\begin{tabular}{|c|c|c|c|c|c|}
\hline $\begin{array}{c}\text { Autor } \\
\text { Ano publicação }\end{array}$ & Objetivo & Método & Resultado & Conclusão & $\begin{array}{l}\text { Escala } \\
\text { PEDro }\end{array}$ \\
\hline $\begin{array}{l}\text { Oh et al. }{ }^{18} \\
2018\end{array}$ & $\begin{array}{l}\text { Avaliar os efeitos da TBA } \\
\text { em indivíduos pós AVC, } \\
\text { quanto a redução da } \\
\text { espasticidade de flexores } \\
\text { plantares e padrão da } \\
\text { marcha }\end{array}$ & $\begin{array}{l}\text { - Ensaio clínico randomizado e } \\
\text { controlado } \\
\text { - Indivíduos com até } 2 \text { anos pós } \\
\text { AVC, hemiparéticos com } \\
\text { espasticidade de flexores } \\
\text { plantares, divididos em } 3 \text { grupos: } \\
\text { - Dentro de } 6 \text { meses ( } n=12 \text { ); } \\
\text { Médio, entre } 6 \text { meses e } 1 \text { ano ( } n= \\
14 \text { ); Tardia, entre } 1 \text { e } 2 \text { anos após } \\
\text { o AVC ( } n=12 \text { ) } \\
\text { - Todos receberam aplicação da } \\
\text { TBA no músculo gastrocnêmio em } \\
\text { pontos da cabeça medial e lateral } \\
\text { com doses determinadas pelo } \\
\text { grau de acometimento individual } \\
\text { - Instrumentos (2, } 4 \text { e } 8 \text { semanas } \\
\text { após aplicação de TBA): MAS, } \\
\text { goniometria e o } 10-T W M\end{array}$ & $\begin{array}{l}\text { - Dos } 40 \text { pacientes iniciais, } \\
38 \text { concluíram o estudo } \\
\text { - Após as } 8 \text { semanas de } \\
\text { aplicação da TBA, os } 3 \\
\text { grupos apresentaram } \\
\text { melhoras significativas em } \\
\text { todos os parâmetros } \\
\text { analisados, exceto o 10- } \\
\text { TWM que não apresentou } \\
\text { aumento da velocidade da } \\
\text { marcha no grupo tardio, } \\
\text { entre } 1 \text { e } 2 \text { anos pós AVC }\end{array}$ & $\begin{array}{l}\text { - O tempo de injeção de TBA } \\
\text { independe da cronicidade do } \\
\text { AVC, e leva uma melhora } \\
\text { consistente da espasticidade } \\
\text { de flexores plantares } \\
\text { mensurados pela MAS, bem } \\
\text { como na ADM de tornozelo } \\
\text { - Não houve redução da } \\
\text { velocidade da marcha, mesmo } \\
\text { naqueles que receberam } \\
\text { injeção precoce e que já } \\
\text { apresentavam marcha } \\
\text { lentificada }\end{array}$ & 9 \\
\hline $\begin{array}{l}\text { Tang et al. }{ }^{23} \\
2012\end{array}$ & $\begin{array}{l}\text { Analisar os efeitos das } \\
\text { injeções de TBA, em } \\
\text { indivíduos pós AVC com } \\
\text { relação a marcha }\end{array}$ & $\begin{array}{l}\text { - Estudo randomizado controlado } \\
\text { duplo cego, com indivíduos pós } \\
\text { AVC, hemiplégicos com padrão } \\
\text { equinovaro sem a necessidade de } \\
\text { utilização de dispositivos } \\
\text { auxiliares para marcha } \\
\text { - Instrumentos: MAS, Fugl-Meyer } \\
\text { e o EMG de superfície coletado } \\
\text { antes e após } 4,8 \text { e } 12 \text { semanas às } \\
\text { injeções de TBA nos pontos } \\
\text { motores dos músculos tríceps } \\
\text { sural, reto femoral e tibial } \\
\text { anterior }\end{array}$ & $\begin{array}{l}\text { - Os escores do MAS foram } \\
\text { reduzidos } \\
\text { significativamente nas } 4,8 \\
\text { e } 12 \text { semanas após } \\
\text { aplicação de TBA, mas os } \\
\text { escores de força não } \\
\text { melhoraram até } 12 \\
\text { semanas após a injeção } \\
\text { - Os padrões EMG } \\
\text { mostraram aumento da } \\
\text { atividade muscular de } \\
\text { todos os músculos que } \\
\text { receberam TBA, sendo } \\
\text { significativa a atividade } \\
\text { dorsiflexora voluntária } \\
\text { ( } p<0,01 \text { ) }\end{array}$ & $\begin{array}{l}\text { - A TBA é eficaz na redução da } \\
\text { ativação muscular antagônica } \\
\text { e promove ativação voluntária } \\
\text { mais funcional dos músculos } \\
\text { dorsiflexores de tornozelo }\end{array}$ & 8 \\
\hline $\begin{array}{l}\text { Lannin et al. }{ }^{24} \\
2018\end{array}$ & $\begin{array}{l}\text { Determinar a adição de } \\
\text { terapia intensiva às } \\
\text { injeções de TBA em } \\
\text { adultos pós AVC sob } \\
\text { enfoque da marcha }\end{array}$ & $\begin{array}{l}\text { - Estudo randomizado controlado } \\
\text { em intenção de tratamento, com } \\
\text { divisão de } 3 \text { grupos de indivíduos. } \\
\text { - Grupo experimental: recebeu } \\
\text { uma dose única de TBA em } \\
\text { músculos, mais } 8 \text { semanas de um } \\
\text { programa de reabilitação } \\
\text { específico (1 hora duas vezes na } \\
\text { semana) } \\
\text { - Grupo controle I: recebeu uma } \\
\text { dose de TBA isoladamente sem a } \\
\text { reabilitação; Grupo controle II: } \\
\text { somente o programa de } \\
\text { reabilitação, sem aplicação de } \\
\text { TBA } \\
\text { - Instrumentos: GAS medidos } \\
\text { após } 8 \text { e } 12 \text { semanas do início das } \\
\text { intervenções }\end{array}$ & $\begin{array}{l}\text { - Embora os participantes } \\
\text { de todos os grupos tenham } \\
\text { aumentado o alcance de } \\
\text { suas metas, o grupo } \\
\text { experimental apresentou } \\
\text { pontuação na GAS maior } \\
\text { em } 10 \% \text { em relação aos } \\
\text { grupos controle após } 12 \\
\text { semanas da intervenção }\end{array}$ & $\begin{array}{l}\text { - O fornecimento de terapia } \\
\text { intensiva após a aplicação de } \\
\text { TBA apresenta melhores } \\
\text { resultados funcionais em } \\
\text { adultos pós AVC }\end{array}$ & 8 \\
\hline $\begin{array}{l}\text { Roche et al. }{ }^{25} \\
2015\end{array}$ & $\begin{array}{l}\text { - Determinar o efeito de } \\
\text { um programa de } \\
\text { reabilitação como um } \\
\text { complemento às injeções } \\
\text { de TBA nas atividades de } \\
\text { marcha em pacientes pós } \\
\text { AVC, hemiparéticos } \\
\text { crônicos }\end{array}$ & $\begin{array}{l}\text { - Estudo randomizado controlado } \\
\text { com distribuição de pacientes } \\
\text { hemiparéticos pós AVC em dois } \\
\text { grupos } \\
\text { - Grupo reabilitação (R): } 19 \\
\text { participantes que receberam TBA } \\
\text { associado a um programa } \\
\text { individual de reabilitação } \\
\text { - Grupo controle (C): com } 16 \\
\text { participantes que receberam } \\
\text { apenas a TBA isoladamente sem o } \\
\text { programa de reabilitação } \\
\text { - Instrumentos: MAS, MRC, } \\
\text { ABILOCO, 10-TWM, TUG, TC6M e } \\
\text { o teste de escada, aplicados após } \\
1 \text { mês da TBA }\end{array}$ & $\begin{array}{l}\text { Em ambos os grupos a } \\
\text { pontuação do MAS } \\
\text { reduziu, em } \\
\text { aproximadamente } 1 \text { ponto } \\
\text { - No grupo } R \text {, houve } \\
\text { aumento da velocidade no } \\
\text { TUG ( } \mathrm{p}=0,01) \text {, ganho de } 1 \% \\
\text { na velocidade durante o } \\
\text { TC6M e de } 9,8 \% \text { na subida } \\
\text { do teste de escada } \\
\text { - No grupo } C \text {, o único } \\
\text { parâmetro que obteve } \\
\text { melhora significativa foi o } \\
\text { tempo de subir e descer } \\
\text { escada }(p=0,04)\end{array}$ & $\begin{array}{l}\text { - A aplicação de TBA em } \\
\text { indivíduos pós AVC possui } \\
\text { eficácia quanto a melhora de } \\
\text { atividades funcionais como a } \\
\text { marcha, porém, ao ser } \\
\text { complementada com um } \\
\text { programa de reabilitação } \\
\text { específico proporciona ganhos } \\
\text { superiores }\end{array}$ & 8 \\
\hline
\end{tabular}




\section{DISCUSSÃO}

A ação da TBA pode ser considerada como um recurso que potencializa a capacidade para andar de indivíduos pós AVC, como o aumento da velocidade, da passada, de distâncias percorridas pelo indivíduo e da redução do tempo de execução de atividades como: subir e descer escadas. ${ }^{23-26}$

$O$ uso da TBA vem sendo amplamente realizado em indivíduos pós AVC, porém, não há consenso na literatura sobre em qual fase da lesão apresenta melhores resultados. ${ }^{27-29}$ Nos estudos desta revisão foi observado que a aplicação da TBA ocorreu comumente na fase crônica do AVC, e segundo os autores, $^{30,31}$ esta fase já apresenta alterações de estrutura e função do corpo instaladas como o enrijecimento tecidual, a contratura e o encurtamento muscular devido a compensações advindas da neuroplasticidade negativa, sendo mais difícil a atuação de abordagens conservadoras, como a fisioterapia, dando preferência por recursos medicamentosos ou cirúrgicos.

$\mathrm{O}$ que de fato não ocorre nas fases iniciais pós AVC, pois os mecanismos de recuperação estão mais críticos que os de compensação, favorecendo a neuroplasticidade positiva, haja visto que a força e função motora estão relacionadas com a reorganização cortical presente nesta fase da doença, como também, as adaptações psicossociais do indivíduo a este novo momento da condição de saúde. ${ }^{27}$

Nesta revisão, foi observado que todos os estudos atenderam os critérios de seleção para aplicação de TBA nos músculos espásticos.

Segundo a American Academy of Neurology Assesment, ${ }^{32}$ os critérios de seleção para aplicação de TBA nos músculos espásticos são: pontos dolorosos e/ou aumento de tônus muscular, encurtamento muscular que reduzem a amplitude de movimento articular, observações quanto às limitações funcionais e análise cinemática da marcha. Entretanto, apenas o profissional médico foi o responsável por determinar qual destes critérios justificaria a aplicação da TBA nos indivíduos pós AVC.

A avaliação multiprofissional com abordagem interdisciplinar permite ser resolutivo e integral no cuidado e neste contexto, agregar outros profissionais de saúde no processo de avaliação para aplicação de TBA é uma estratégia ${ }^{33}$ que pode facilitar este processo, ${ }^{34}$ além da participação do indivíduo e ou família, possibilitando o alcance de melhores resultados no tratamento. ${ }^{35}$

Quanto ao grupo muscular para receber a TBA, esta revisão apontou que os flexores plantares (gastrocnêmio e sóleo) foram os músculos preferencialmente escolhidos. Sabe-se que, ocorre maior frequência de espasticidade neste grupo muscular pós AVC por ter ação postural e a aplicação da TBA pode melhorar na geração de torque durante a fase da marcha, favorecendo a impulsão anterior e aumento na velocidade da atividade de andar. ${ }^{36}$

De acordo com a análise biomecânica da marcha realizada por Novaes et al..$^{35}$ os flexores plantares iniciam uma contração concêntrica que leva o membro inferior à fase de balanço, correspondendo a $80 \%$ do ciclo da marcha, gerando a maior parte da energia mecânica.

Dessa forma, otimizar o aumento da velocidade da marcha pode impactar na capacidade para andar de indivíduos pós
AVC, pois melhora esta ação no contexto do dia a dia. ${ }^{30,31}$

Para avaliação da intervenção existem vários instrumentos que testam a capacidade da atividade andar aplicáveis e recomendados para indivíduos pós $A V C .{ }^{26} \mathrm{Na}$ presente revisão ${ }^{23-26}$ para avaliar a resposta da TBA foram descritas ferramentas como a Escala Modificada de Ashwoth (MAS), a Escala de Fulg Meyer, o Teste de caminhada de 6 minutos (TC6M), o Time Up and Go (TUG), o Teste do degrau para avaliar tríplice flexão de MMII, a Escala de Realização de Objetivos (GAS) e o teste de caminhada de 10 metros (10-TWM). ${ }^{23,26}$

Dentre os instrumentos citados e os que atingem o objetivo de avaliar capacidade da atividade de andar, o teste de caminhada de 10 metros (10-TWM) foi o mais utilizado. Este teste permite avaliar o tempo gasto para que o paciente percorra 10 metros em sua velocidade máxima de caminhada e possibilita a contagem de passos para determinar a cadência da marcha, permitindo assim a análise da velocidade de caminhada. ${ }^{37}$ Os estudos que utilizaram este instrumento colocam que é um teste simples e que permite identificar o grau de capacidade quanto a atividade de andar em indivíduos pós AVC, antes e pós intervenção. ${ }^{23,26}$

Por fim, os estudos selecionados para análise final desta revisão foram avaliados quanto à qualidade pela escala PEDro, porém, observou-se pela avaliação dos autores que itens quanto ausência de alocação dos participantes cega/secreta, ${ }^{26}$ como também dos terapeutas ${ }^{25,26}$ não foram atendidos, mas justificado pelo tipo de intervenção (injeção muscular). Além disso, todos os estudos aqui analisados avaliaram o grau de melhora da atividade de andar em até 12 semanas, que é o período de ação da TBA, o que limita afirmar que estes benefícios perduram a longo prazo. Entretanto, a identificação destes benefícios mostra o quão é importante a educação em saúde para o paciente e seus familiares.

Isto é, esclarecer que há benefícios da TBA, porém, é essencial utilizar este período para adaptar-se, aproveitando as facilitações que a toxina traz, e principalmente, modificar padrões compensatórios e comportamentos sedentários que corroboram para o retorno das limitações da atividade e restrições de desempenho e não devido ao término da ação da toxina. Deste modo, visando ampliar a análise do efeito da TBA sobre a marcha, sugere-se estudos longitudinais com maior tempo de acompanhamento dos pacientes.

O presente manuscrito discorre sobre uma revisão sistemática e este tipo de delineamento está sujeito a algumas desvantagens, como aqueles relacionados à viés seleção. Embora possa ser considerado como uma limitação no presente estudo, a fim de minimizar os erros foram adotadas estratégias para garantir o rigor científico, dada a importância reconhecida de revisões sistemáticas.

Assim, neste trabalho o processo de extração de dados foi realizado de acordo com os critérios de inclusão e exclusão adotados por dois revisores, que coletaram as informações dos estudos de forma independente e resolvendo as discordâncias por meio de um consenso.

Além disso, os estudos incluídos foram avaliados pela escala PEDro, garantindo uma qualidade metodológica comparável entre si. Entretanto, o número reduzido de estudos aptos a esta revisão infere a necessidade de maiores aprofundamentos sobre o uso da TBA nesses indivíduos. 


\section{CONCLUSÃO}

Considerando os estudos analisados e classificados como alta e moderada qualidade da evidência de acordo com a escala PEDro foi observado que, a TBA proporciona o aumento da passada, da velocidade e das distâncias percorridas pelo indivíduo, reduz o tempo de execução de atividades como: subir e descer escadas em indivíduos pós AVC. A identificação destes benefícios reforça que é essencial educar os pacientes, da importância de aproveitar este momento para modificar comportamentos que acentuam os padrões de compensação que corroboram para o retorno de limitações de atividades, e não devido ao término da ação da TBA.

\section{REFERÊNCIAS}

1. Organização Mundial da Saúde. 10 principais causas de morte no mundo [texto na internet]. Brasília: OPAS; c2020 [citado 2020 Jun 23]. Disponível em: https://www.paho.org/bra/

2. World Health Organization. Health statistics and information systems - Projections of mortality and causes of death, 2015 and 2030 [text on the Internet]. Geneva: WHO; c2013. [cited 2020 jun 23]. Available from: https://www.who.int/healthinfo/global_burden_disease/pr ojections2015 2030/en/

3. Damata SRR, Formiga LMF, Araújo AKS, Oliveira EAR, Oliveira AKS, Formiga RCF. Perfil epidemiológico dos idosos acometidos por acidente vascular cerebral. Rev Interd. 2016;9(1):107-17.

4. Gittler M, Davis AM. Guidelines for Adult Stroke Rehabilitation and Recovery. JAMA. 2018;319(8):820-1. Doi: https://doi.org/10.1001/jama.2017.22036

5. Francisco SEC. Modelos de intervenção em Fisioterapia nos pacientes com espasticidade pós AVC: revisão da literatura [Dissertação]. Lisboa: Instituto Politécnico de Lisboa; 2016.

6. Phadke CP, Ismail F, Boulias C, Gage W, Mochizuki G. The impact of post-stroke spasticity and botulinum toxin on standing balance: a systematic review. Expert Rev Neurother. 2014;14(3):319-27.

Doi: https://doi.org/10.1586/14737175.2014.887443

7. Williams SA, Reid S, Elliott C, Shipman P, Valentine J. Muscle volume alterations in spastic muscles immediately following botulinum toxin type-A treatment in children with cerebral palsy. Dev Med Child Neurol. 2013;55(9):813-20.

Doi: https://doi.org/10.1111/dmcn.12200

8. Farias N, Buchalla CM. A Classificação Internacional de Funcionalidade, Incapacidade e Saúde da Organização Mundial da Saúde: conceitos, usos e perspectivas. Rev Bras Epidemiol. 2005;8(2):187-93. Doi: http://dx.doi.org/10.1590/S1415-790X2005000200011

9. Schinwelski MJ, Sitek EJ, Wąż P, Sławek JW. Prevalence and predictors of post-stroke spasticity and its impact on daily living and quality of life. Neurol Neurochir Pol. 2019;53(6):449-57.

Doi: https://doi.org/10.5603/PJNNS.a2019.0067
10. Datta Gupta A, Visvanathan R, Cameron I, Koblar SA, Howell S, Wilson D. Efficacy of botulinum toxin in modifying spasticity to improve walking and quality of life in post-stroke lower limb spasticity - a randomized double-blind placebo controlled study. BMC Neurol. 2019;19(1):96. Doi: https://doi.org/10.1186/s12883-0191325-3

11. Sheean G, Lannin NA, Turner-Stokes L, Rawicki B, Snow BJ; Cerebral Palsy Institute. Botulinum toxin assessment, intervention and after-care for upper limb hypertonicity in adults: international consensus statement. Eur J Neurol. 2010;17 Suppl 2:74-93. Doi: https://doi.org/10.1111/j.1468-1331.2010.03129.x

12. Olver J, Esquenazi A, Fung VS, Singer $B J$, Ward $A B$; Cerebral Palsy Institute. Botulinum toxin assessment, intervention and aftercare for lower limb disorders of movement and muscle tone in adults: international consensus statement. Eur J Neurol. 2010;17 Suppl 2:5773. Doi: https://doi.org/10.1111/j.14681331.2010.03128.x

13. Sun LC, Chen R, Fu C, Chen Y, Wu Q, Chen R, et al. Efficacy and safety of Botulinum toxin type $A$ for limb spasticity after stroke: a meta-analysis of randomized controlled trials. Biomed Res Int. 2019;2019:8329306. Doi: https://doi.org/10.1155/2019/8329306

14. Picelli A, Santamato A, Chemello E, Cinone N, Cisari C, Gandolfi M, et al. Adjuvant treatments associated with botulinum toxin injection for managing spasticity: An overview of the literature. Ann Phys Rehabil Med. 2019;62(4):291-6.

Doi: https://doi.org/10.1016/i.rehab.2018.08.004

15. Park J, Chung ME. Botulinum toxin for central neuropathic pain. Toxins (Basel). 2018;10(6):224. Doi: https://doi.org/10.3390/toxins10060224

16. Yan X, Lan J, Liu Y, Miao J. Efficacy and Safety of Botulinum Toxin Type A in Spasticity Caused by Spinal Cord Injury: A Randomized, Controlled Trial. Med Sci Monit. 2018;24:8160-71. https://doi.org/10.12659/MSM.911296

17. Nasb M, Li Z, Youssef ASA, Dayoub L, Chen H. Comparison of the effects of modified constraint-induced movement therapy and intensive conventional therapy with a botulinum-a toxin injection on upper limb motor function recovery in patients with stroke. Libyan J Med. 2019;14(1):1609304. https://doi.org/10.1080/19932820.2019.1609304

18. Oh HM, Park GY, Choi YM, Koo HJ, Jang Y, Im S. The effects of Botulinum toxin injections on plantar flexor spasticity in different phases after stroke: a secondary analysis from a double-blind, randomized trial. PM R. 2018;10(8):78997. Doi: https://doi.org/10.1016/j.pmrj.2018.02.011

19. Karadag-Saygi E, Cubukcu-Aydoseli K, Kablan N, Ofluoglu D. The role of kinesiotaping combined with botulinum toxin to reduce plantar flexors spasticity after stroke. Top Stroke Rehabil. 2010;17(4):318-22. Doi: https://doi.org/10.1310/tsr1704-318 
20. Schinwelski M, Sławek J. Prevalence of spasticity following stroke and its impact on quality of life with emphasis on disability in activities of daily living. Systematic review. Neurol Neurochir Pol. 2010;44(4):404-11. Doi: https://doi.org/10.1016/s0028-3843(14)60300-5

21. Santos CMC, Pimenta CAM, Nobre MRC. The PICO strategy for the research question construction and evidence search. Rev Lat Am Enfermagem. 2007;15(3):508-11. Doi: https://doi.org/10.1590/s0104$\underline{11692007000300023}$

22. Shiwa RS, Costa LOP, Moser ADL, Aguiar IC, Oliveira LVF. PEDro: a base de dados de evidências em fisioterapia. Fisioter Mov. 2011;24(3):523-33. Doi: https://doi.org/10.1590/S0103-51502011000300017

23. Tang SF, Hong JP, McKay WB, Tang CW, Wu PH, Chu NK. Modification of altered ankle motor control after stroke using focal application of botulinum toxin type A. Clin Neurol Neurosurg. 2012;114(5):498-501. Doi: https://doi.org/10.1016/i.clineuro.2012.03.014

24. Lannin NA, Ada L, Levy T, English C, Ratcliffe J, Sindhusake $D$, et al. Intensive therapy after botulinum toxin in adults with spasticity after stroke versus botulinum toxin alone or therapy alone: a pilot, feasibility randomized trial. Pilot Feasibility Stud. 2018;4:82.

Doi:

https://doi.org/10.1186/s40814-018-0276-6

25. Roche N, Zory R, Sauthier A, Bonnyaud C, Pradon D, Bensmail D. Effect of rehabilitation and botulinum toxin injection on gait in chronic stroke patients: a randomized controlled study. J Rehabil Med. 2015;47(1):31-7. Doi: https://doi.org/10.2340/16501977-1887

26. Marinho C, Monteiro M, Santos L, Oliveira-Filho J, Pinto EB. Desempenho da marcha e qualidade de vida nos sobreviventes de avc: um estudo transversal. Rev Pesq Fisio. 2018;8(1):79-87. Doi: http://dx.doi.org/10.17267/2238-2704rpf.v8i1.1777

27. Li S. Spasticity, Motor Recovery, and Neural Plasticity after Stroke. Front Neurol. 2017;8:120. Doi: https://doi.org/10.3389/fneur.2017.00120

28. Maki T. Toxina botulínica tipo A e estimulação elétrica funcional no membro superior de pacientes crônicos pósacidente vascular cerebral [Dissertação]. Campinas: Universidade Estadual de Campinas; 2005.
29. Zilli F, Lima ECBA, Kohler MC. Neuroplasticidade na reabilitação de pacientes acometidos por AVC espástico. Rev Ter Ocup Univ São Paulo. 2014;25(3):317-22. Doi: https://doi.org/10.11606/issn.2238-6149.v25i3p317-322

30. Simão SSS, Romero VU, Baraldil K, Oda AL, Viana CF, Chiappetta ALML, et al. Avaliação clínica da relação entre postura, respiração e deglutição em paciente pósacidente vascular cerebral na fase crônica: relato de caso. Rev CEFAC. 2013; 15 (5):1371-8. Doi: https://doi.org/10.1590/S1516-18462013000500035

31. Dunne JW, Gracies JM, Hayes M, Zeman B, Singer BJ; Multicentre Study Group. A prospective, multicentre, randomized, double-blind, placebo-controlled trial of onabotulinumtoxinA to treat plantarflexor/invertor overactivity after stroke. Clin Rehabil. 2012;26(9):787-97. Doi: https://doi.org/10.1177/0269215511432016

32. Tao W, Yan D, Li JH, Shi ZH. Gait improvement by low-dose botulinum toxin $A$ injection treatment of the lower limbs in subacute stroke patients. J Phys Ther Sci. 2015;27(3):759-62. https://doi.org/10.1589/ipts.27.759

33. Souza A, Tavares JMRS. A marcha humana: uma abordagem biomecânica. UCH Gaia-Porto. 2010;1:1-9.

34. Ricci NA, Ferrarias GP, Molina KI, Dib PM, Alouche SR. Velocidade de marcha e autoeficácia em quedas em indivíduos com hemiparesia após Acidente Vascular Encefálico. Fisioter Pesq. 2015;22(2):191-6. Doi: https://doi.org/10.590/1809-2950/14484522022015

35. Novaes RD, Miranda AS, Dourado VZ. Velocidade usual da marcha em brasileiros de meia idade e idosos. Rev Bras Fisioter. 2011;15(2):117-22. Doi: https://doi.org/10.1590/S1413-35552011000200006

36. Nascimento LR, Caetano LCG, Freitas DCMA, Morais TM, Polese JC, Teixeira-Salmela LF. Diferentes instruções durante teste de velocidade de marcha determinam aumento significativo na velocidade máxima de indivíduos com hemiparesia crônica. Rev Bras Fisioter. 2012;16(2):122-7. Doi: https://doi.org/10.1590/S141335552012005000008 